\title{
Wait times hold steady but vary by region
}

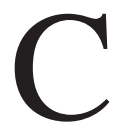

anada's health system is keeping up with greater demand for priority surgeries, but wait times still vary widely across the country, even between similar health regions, the Canadian Institute for Health Information (CIHI) reports.

Every year since 2010 , about $80 \%$ of patients have had hip and knee replacements, hip fracture repairs and cataract surgery within recommended times, while $98 \%$ received timely radiation therapy, according to Wait Times for Priority Procedures in Canada, 2015. Wait times have been stable, even as demand for these surgeries has grown. For example, in the past five years, the number of radiation treatments has increased by $34 \%$; hip and knee replacements increased by $28 \%$ and $24 \%$, respectively.

The new data suggest that health providers are delivering more efficient care, says Tracy Johnson, manager of emerging issues at CIHI. "They're coping at the moment with the load and the increased number of patients."

Canada is also performing well compared to six international peers with similar public health systems: the United Kingdom, Australia, New Zealand, Finland, Portugal and Estonia. Of these countries, Canada has the shortest wait times for cataract surgery, and second shortest waits after the UK for hip and knee replacements.

“Often Canada doesn't do well in international comparisons of wait times for primary and specialist care, and we spend a lot of time wondering if our benchmarks are appropriate and if we're seeing enough patients," says Johnson. "But this shows that when we focus in and have something to measure, we've done well in Canada."

Despite this sunny national portrait, the data paint a less optimistic picture of access at the regional level. For the first time, CIHI compared surgical wait times between health regions and found wide variations in delays to treatment.

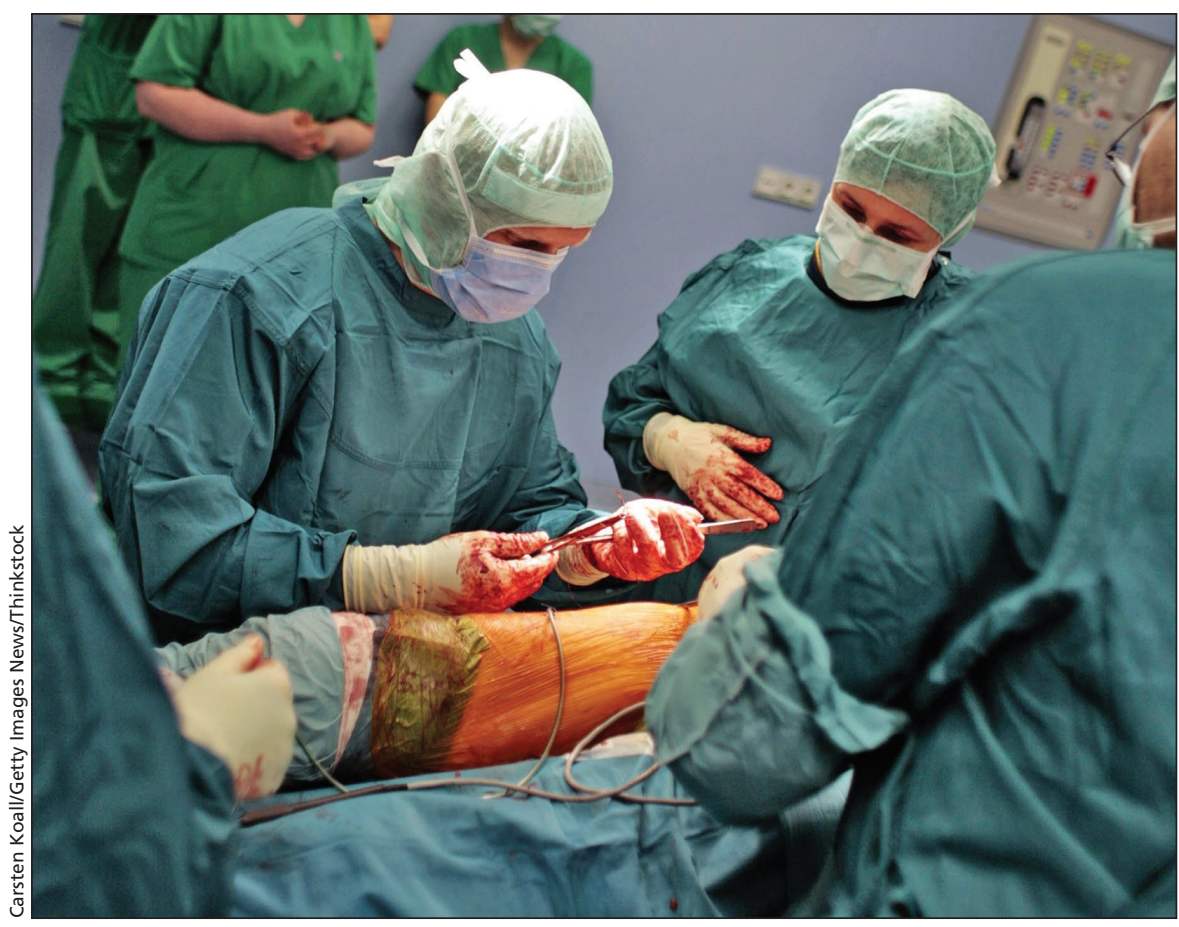

Wait times for priority surgeries have been stable over the past five years.

For example, in the six Ontario local health integration networks serving Toronto and its surrounding areas, the proportion of patients receiving care within benchmarks varied from $54 \%$ to $98 \%$ for hip replacements, and $50 \%$ to $96 \%$ for knee replacements.

Such variation between otherwise similar health regions isn't easily explained away by population density or geographic remoteness, says Johnson. "There doesn't appear to be a pattern; one would think that when you're looking at similar populations you would see similar patient flows, but you don't, which suggests that there are probably some lessons to be learned."

And Ontario isn't the only province with regional variations in wait times, says Johnson. "Aside from New Brunswick, Newfoundland and Saskatchewan, most of the provinces have a wide variation." Possibly these outlier provinces have fewer regions providing the surgeries CIHI studied. "Newfoundland has also had a focus on improving their joints and Saskatchewan has had an overall focus on their surgical initiative in the past couple of years, which has improved their waits considerably," adds Johnson.

$\mathrm{CIHI}$ also reported wide variations in wait times for hip fracture repairs in the Yukon depending on whether a patient was sent out of the territory for surgery. Wait times were comparable to the rest of Canada for patients requiring hip fracture repair who underwent the procedure locally, with $80 \%$ receiving treatment within the recommended 48 hours. In comparison, just $31 \%$ of those who had to travel to nearby provinces for the surgery received treatment within the benchmark.

Johnson says that a big part of the problem appears to be delays in air travel. "Medical evacuation can take 6-12 hours, and there's no night flying, so people have to wait for an opportunity to fly out the next morning." Lauren Vogel, CMAJ

CMAJ 2015. DOI:10.1503/cmaj.109-5042 